\title{
Radiative $\phi$ decays with derivative interactions
}

\author{
Francesco Giacosa and Giuseppe Pagliara \\ Institut für Theoretische Physik, Johann Wolfgang Goethe University, \\ Max-von-Laue-Str. 1, D-60438 Frankfurt, Germany
}

\begin{abstract}
We study the line shapes of radiative $\phi$-decays involving virtual $f_{0}(980)$ and $a_{0}(980)$ mesons which decay, via derivative couplings, to $\pi^{0} \pi^{0}$ and $\pi^{0} \eta$ respectively. After developing the formalism for derivative interactions at one-loop level, we show that they can reproduce the measured peaked line shapes of $\phi$-decays without including kaon loops.
\end{abstract}

\section{INTRODUCTION}

The radiative decays of the $\phi$ meson are a valuable tool to study the nature of light scalar states below $1 \mathrm{GeV}$ [1, 2, 3]. It is still debated if the latter are quarkonia [4], tetraquark [5, 6], molecular states [7] or a mixing of these configurations [8, 9]. A dominant quarkonium assignment is problematic [10], thus leading to identify the $\bar{q} q$-states with resonances above $1 \mathrm{GeV}$ where mixing with the scalar glueball takes place [11, 12].

In this article we do not employ a particular interpretation for the resonances $f_{0} \equiv f_{0}(980)$ and $a_{0} \equiv a_{0}(980)$. Independently on their nature, spontaneous breaking of chiral symmetry implies that derivative couplings to the pseudoscalar mesons $\pi, \eta$ and $K$ arise. In fact, the latter states are the emerging Goldstone bosons, which can be rewritten as angular excitations. Then, Lagrangian interactions of derivative type with schematic form $\mathcal{L}_{\text {int }} \sim$ $S\left(\partial_{\mu} \varphi_{1}\right)\left(\partial^{\mu} \varphi_{2}\right)$ with $S=f_{0}, a_{0}$ and $\varphi_{1,2}=\pi, \eta, K$, are obtained [13]. Our aim is to study in detail the effects of such derivative interactions on the line shapes of the reactions $\phi \rightarrow \gamma \pi^{0} \pi^{0}$ and $\phi \rightarrow \gamma \pi^{0} \eta$, occurring via virtual $f_{0}$ and $a_{0}$ mesons respectively.

In order to discuss properly the issue we concentrate on the reaction $\phi \rightarrow \gamma \pi^{0} \pi^{0}$ via a virtual $f_{0}$ state. We first write down explicitly the general interaction Lagrangian of the $f_{0}$ meson with pions and kaons as emerging upon chiral symmetry breaking within the nonlinear realization of chiral symmetry as obtained in Ref. [13]:

$$
\mathcal{L}_{i n t, f_{0}}=c_{f_{0} \pi \pi} f_{0}\left(\partial_{\mu} \vec{\pi}\right)^{2}+d_{f_{0} \pi \pi} M_{\pi}^{2} f_{0} \vec{\pi}^{2}+c_{f_{0} K K} f_{0}\left(\partial_{\mu} K^{+}\right)\left(\partial^{\mu} K^{-}\right)+d_{f_{0} K K} M_{K}^{2} f_{0}\left(K^{+} K^{-}\right)+\ldots
$$

where dots refer to the analogous terms with the neutral kaon states. In the chiral limit the pion and the kaon masses $M_{\pi}$ and $M_{K}$ vanish leaving only interactions with derivatives parametrized by the coupling constants $c_{f_{0} \pi \pi}$ and $c_{f_{0} K K}$. The constants $d_{f_{0} \pi \pi}$ and $d_{f_{0} K K}$ parametrize the interaction without derivatives. Now, the radiative decay $\phi \rightarrow \gamma \pi^{0} \pi^{0}$ via $f_{0}$ can occur essentially in two ways:

(a) An interaction Lagrangian for the decay $\phi \rightarrow \gamma f_{0}$ is introduced as $\mathcal{L}_{\phi \gamma f_{0}}=c_{\phi \gamma f_{0}} f_{0} F^{\mu \nu} V_{\mu \nu}$, where $F^{\mu \nu}=$ $\partial^{\mu} A^{\nu}-\partial^{\nu} A^{\mu}$ is the electromagnetic field strength and $V_{\mu \nu}=\partial_{\mu} \phi_{\nu}-\partial_{\nu} \phi_{\mu}$ is the field strength related to the vector field $\phi_{\mu}$. The interaction Lagrangian $\mathcal{L}_{\phi \gamma f_{0}}$, parameterized by $c_{\phi \gamma f_{0}}$, corresponds to a point-like coupling which effectively takes into account loops of quarks (whose precise form depends on the microscopic interpretation of the $f_{0}$ meson). After the transition $\phi \rightarrow \gamma f_{0}$ the scalar meson $f_{0}$ decays into pions (as depicted in Fig. 1.c) via derivative couplings (mechanism a.1, whose amplitude is proportional to $c_{f_{0} \pi \pi}$ ) or via non-derivative couplings (mechanism a.2, proportional to $d_{f_{0} \pi \pi}$ ). A first study of derivative interactions (thus setting $d_{f_{0} \pi \pi}=d_{f_{0} K K}=0$ ) has been performed in Ref. [14], where it has been shown by using Breit-Wigner propagators for the scalars that derivative couplings describe the data better than non-derivative ones. A detailed study involving non-derivative interactions only (setting $\left.c_{f_{0} \pi \pi}=c_{f_{0} K K}=0\right)$ has been performed in Ref. [15].

(b) The vector meson $\phi$ couples strongly to kaons. Then, via a kaon-loop a photon is generated at one vertex and a $f_{0}$ meson at the other vertex. The latter coupling can again occur in two ways: via derivative coupling (mechanism b.1, proportional to $c_{f_{0} K K}$, see Ref. [14]) or via non derivative coupling (mechanism b.2, proportional to $d_{f_{0} K K}$, see Ref. [2, 16, 17]).

Note that all these considerations apply also for the $a_{0}$ meson as a virtual state of the reaction $\phi \rightarrow \gamma \pi^{0} \eta$ : the derivative (non-derivative) interactions with $\pi \eta$ and $\bar{K} K$ are parameterized by $c_{a_{0} \pi \eta}\left(d_{a_{0} \pi \eta}\right)$ and $c_{a_{0} K K}\left(d_{a_{0} K K}\right)$ in a Lagrangian which is analogous to Eq. (11). Clearly, the possible decay mechanisms are also separated into a.1 and a.2 (direct, non-structure coupling) and via kaon loops (b.1 and b.2).

It is evident that the description of the radiative decay $\phi \rightarrow \gamma \pi^{0} \pi^{0}$ (and $\phi \rightarrow \gamma \pi^{0} \eta$ ) is difficult because four decay mechanisms (a.1, a.2 and b.1, b.2) can potentially contribute and it is not clear a priori which one is dominant. As a consequence, considering that the inclusion of all contributions at the same time has not yet been performed, the extraction of the parameters of Eq. (1) from experiments depends also on theoretical assumptions.

Mechanism (b) is a mesonic 1-loop contribution to the decay mechanism, which is regarded as dominant by many authors in view of the large coupling to kaons of the vector meson $\phi$ meson and of the scalars $a_{0}(980)$ and $f_{0}(980)$. 
However, notice that the mechanism (a) is dominant according to large- $\mathrm{N}_{c}$ counting rule both in the tetraquark and quarkonium assignments for the $f_{0}(980)$ meson and in the tetraquark assignment for the $a_{0}(980)$ [18] (see also the note to this Reference). While it is not yet clear to which extent large- $\mathrm{N}_{c}$ is reliable in this context -see also Ref. [3] for a discussion of this point-, we consider this fact as a motivation to study in detail the effects of the mechanism (a) on radiative decays and thus to test an alternative scenario for the description of radiative $\phi$ decays. Moreover, in this work we restrict to the chiral limit dominant mechanism a.1 where only derivatives are involved (corresponding to setting $d_{f_{0} \pi \pi}=d_{f_{0} K K}=d_{a_{0} \pi \eta}=d_{a_{0} K K}=0$ ). We thus intend to continue the analysis initiated in Ref. [14] about derivative interactions by studying the decay mechanism a. 1 in both the $f_{0}$ and the $a_{0}$ channels in relation to radiative $\phi$ decays. We aim to do it by properly taking into account loops and finite-width effects using the formalism developed in Ref. [19] extended to the case of derivative couplings. That is, both real and imaginary parts of selfenergy contributions (which show a rather different behavior than their non-derivative counterparts, see Fig. 2) are taken into account. We regularize the model by using an effective cutoff of the order of $1 \mathrm{GeV}$ which is introduced by using a nonlocal extension of Eq. (11). We conclude this discussion by noting that the neglect of the non-derivative coupling is surely justified in virtue of the small pion mass in the $\pi \pi$ channel of $f_{0}$ but is less justified in $\pi \eta$ channel of $a_{0}$ and in the kaon-kaon channel of both resonances because of the larger masses of the $K$ and $\eta$ mesons. The next step shall be the inclusions of mechanism a.2. but at the present stage a fit with non-zero $c$ 's and $d$ 's at the same time would not be constrained enough.

Quite remarkably, our analysis shows that derivative interactions alone work well in the description of both $\phi \rightarrow$ $\gamma \pi^{0} \pi^{0}$ and $\phi \rightarrow \gamma \pi^{0} \eta$ line shapes as experimentally measured in the SND and KLOE collaborations in Refs. [20, 21] for the $f_{0}$ meson and in Refs. 222, 23] for the $a_{0}$ meson. The peaked line shapes can be reproduced in virtue of the derivative coupling which enhance the theoretical curves close to threshold.

We also compare our results with Ref. [24], where the $f_{0}$ meson has been studied studied in $j / \psi$ decays at BES, and with Ref. 25], in which an experimental analysis of the Crystal Barrel data for the $a_{0}$ meson has been performed, see also Refs. [26, 27]. However, we shall also point out that care is needed when extracting the coupling constants of scalar-to-pseudoscalar from radiative $\phi$ decays alone because of strong correlations of the parameters entering in the fit. In particular, the difficulty is due to the the fact that, if the decay mechanism (a) is dominant, an extraction of $c_{f_{0} K K}$ (and eventually $d_{f_{0} K K}$ ) from the line shapes of $\phi \rightarrow \gamma \pi^{0} \pi^{0}$ reaction is hard because $c_{f_{0} K K}$ and $d_{f_{0} K K}$ appear only in the denominator of the propagator of $f_{0}$ and are not directly proportional to decay amplitude(s). (This is not the case for mechanism $\mathrm{b}$, where the kaon-loop amplitudes are directly proportional to $c_{f_{0} K K}$ and $d_{f_{0} K K}$ ). A similar discussion holds in the $a_{0}$ case. For this reason we did not include the coupling to kaons as free parameter of the fit but we repeated the analysis for different values of the latter checking how the fit is affected.

As a comparison we also perform the fit using non-derivative couplings only (that is we set $c_{f_{0} \pi \pi}=c_{f_{0} K K}=0$ and we leave $d_{f_{0} \pi \pi}$ and $d_{f_{0} K K}$ free) and we show that a bad description of data for the $f_{0}$ meson is obtained. In the $a_{0}$ case $\left(c_{a_{0} \pi \eta}=c_{a_{0} K K}=0\right.$ and $d_{a_{0} \pi \eta}$ and $d_{a_{0} K K}$ free), while a fit without derivatives is still acceptable, the resulting parameters are not compatible with the experimental results of Ref. [25]. Thus, the need of including derivative-like couplings is favoured by our study.

The paper is organized as follows: in the next Section we extend the formalism of [19] to the derivative interaction and we derive the theoretical expressions for the lines shapes of $\phi$-decays. In the third Section we present the fits to the experimental results and we study the correlation of the parameters. In the fourth Section we drive our conclusions and outlook.

\section{II. $\phi$ DECAYS WITHIN DERIVATIVE INTERACTIONS}

\section{A. 1-Loop within derivative interactions}

We generalize the study of Ref. [19] to which we refer for a careful treatment of the definitions and relative discussions, by considering the following nonlocal interaction Lagrangian of derivative type:

$$
\mathcal{L}=\frac{1}{2}\left(\partial_{\mu} S\right)^{2}-\frac{1}{2} M_{0}^{2} S^{2}+\frac{1}{2}\left(\partial_{\mu} \varphi\right)^{2}-\frac{1}{2} m^{2} \varphi^{2}+\mathcal{L}_{\text {int }} ; \mathcal{L}_{\text {int }}=g S(x) \int \mathrm{d}^{4} \mathrm{y} \partial_{\mu} \varphi(x+y / 2) \partial^{\mu} \varphi(x-y / 2) \Phi(y)
$$

with $\partial_{\mu}=\partial / \partial x^{\mu}$. The nonlocality describes the finite dimensions of the scalar states [19]: it takes into account already at the Lagrangian level of a form factor in the expression of the decay width. Previous studies (see, for instance, Refs. [11, 28] and also the more microscopic approach of Ref. [29]) show that a cut-off of the order of $1 \mathrm{GeV}$ emerges in the context of phenomenological mesonic theories. By introducing the function $f_{\Lambda}(q)$ as the Fourier transform of $\Phi(y), f_{\Lambda}(q)=\int d^{4} y \Phi(y) e^{-i y q}$, the tree-level Feynman amplitude of Fig. 11a reads -ig $\left(q_{1} \cdot q_{2}\right) f_{\Lambda}\left(\left(q_{1}-q_{2}\right) / 2\right)$ where $q_{1}$ and $q_{2}$ are the momenta of the two particles $\varphi$. Let $p=q_{1}+q_{2}$ be the momentum of the particle $S$. 


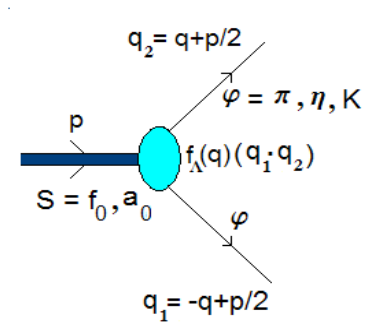

(a) Nonlocal derivative vertex

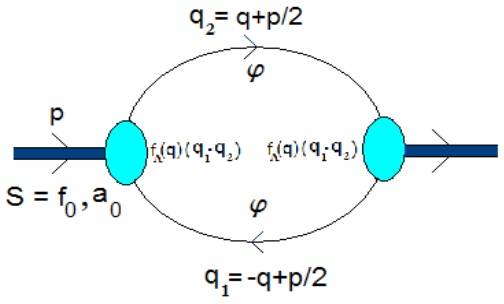

(b) Self-energy

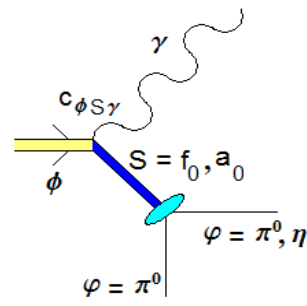

(c) Radiative $\phi$-decay

FIG. 1: Relevant Feynman diagrams [colors online].

The decay width is evaluated in the reference frame of $S$, in which we have:

$$
p=\left(M_{0}, \overrightarrow{0}\right) ; q_{1}=\left(\sqrt{\vec{q}^{2}+m^{2}}, \vec{q}\right) ; q_{2}=\left(\sqrt{\vec{q}^{2}+m^{2}},-\vec{q}\right) ; q_{\varphi \varphi}\left(M_{0}\right)=|\vec{q}|=\sqrt{\frac{M_{0}^{2}}{4}-m^{2}} .
$$

The tree-level decay reads explicitly:

$$
\Gamma_{S \varphi \varphi}^{\mathrm{t}-1}\left(M_{0}\right)=\frac{q_{\varphi \varphi}\left(M_{0}\right)}{8 \pi M_{0}^{2}}\left[(\sqrt{2} g)\left(\frac{M_{0}^{2}-2 m^{2}}{2}\right) f_{\Lambda}(0, \vec{q})\right]^{2} \theta\left(M_{0}-2 m\right)
$$

where the equality $q_{1} \cdot q_{2}=\left(M_{0}^{2}-2 m^{2}\right) / 2$ has been used. The tree-level propagator $\Delta_{S}(p)=\left[p^{2}-M_{0}^{2}+i \varepsilon\right]^{-1}$, valid in the limit $g \rightarrow 0$, is modified by the 1-loop correction and takes the form $\Delta_{S}\left(x=\sqrt{p^{2}}\right)=$ $\left[x^{2}-M_{0}^{2}+(\sqrt{2} g)^{2} \Sigma(x, m)+i \varepsilon\right]^{-1}$ in which the self-energy $\Sigma(x, m)$, see Fig. 1 b, is given by:

$$
\Sigma\left(x=\sqrt{p^{2}}, m\right)=-i \int \frac{d^{4} q}{(2 \pi)^{4}} \frac{\left[\left(q_{1} \cdot q_{2}\right) f_{\Lambda}\left(q^{0}, \vec{q}\right)\right]^{2}}{\left[q_{1}^{2}-m^{2}+i \varepsilon\right]\left[q_{2}^{2}-m^{2}+i \varepsilon\right]}
$$

with $q_{1}=q+p / 2$ and $q_{2}=-q+p / 2$. For future convenience we consider the loop as a function of $x=\sqrt{p^{2}}$ and of the mass $m$. Note that the only difference with respect to the non-derivative study of Ref. [19] is the extra-factor $\left(q_{1} \cdot q_{2}\right)^{2}$ in the tree-level decay rate and in the numerator of the self energy.

A possible choice of a three-dimensional cutoff, which makes the model finite, corresponds to $\left([11,28\right.$, , 29] $) f_{\Lambda}(q)=$ $f_{\Lambda}\left(\vec{q}^{2}\right)=\exp \left[-\vec{q}^{2} / \Lambda^{2}\right]$ with $q_{\varphi \varphi}(x)=\sqrt{\vec{q}^{2}}$ as in Eq. (3). The precise form of the cutoff function does not influence the results as long as convergence is achieved. Moreover, despite the fact that the superficial degree of divergence of the integral in Eq. (5) in the limit of large $\Lambda$ is four, it turns out that the Eq. (5) is only linear divergent in $\Lambda$. In our work we use $\Lambda=1.5 \mathrm{GeV}$, which is very close to the value used in Refs. [11, 29]. Variations of the latter between 1-2 $\mathrm{GeV}$ affects only slightly the results.

A general property for $\Sigma\left(p^{2}\right)$ follows from the optical theorem:

$$
I_{S}(x)=(\sqrt{2} g)^{2} \operatorname{Im}[\Sigma(x, m)]=x \Gamma_{S \varphi \varphi}^{\mathrm{t}-1}(x) .
$$

The imaginary part of the self-energy diagram is zero for $0<x<2 m$ and nonzero starting at threshold. The real part $R_{S}(x)=(\sqrt{2} g)^{2} \operatorname{Re}[\Sigma(x, m)]$ is nonzero below and above threshold. In Fig. 2 the functions $\operatorname{Re}[\Sigma(x, m)]$ and $\operatorname{Im}[\Sigma(x, m)]$ are plotted as an illustrative example.

We define the (nominal) mass $M_{S}$ for the scalar field $S$ as the solution of the equation $M_{S}^{2}-M_{0}^{2}+R_{S}\left(M_{S}\right)=0$. When the function $R\left(M_{S}\right)$ is positive, which is usually the physical case (Fig. 2), the dressed mass $M_{S}$ is smaller than the bare mass $M_{0}$, showing that the quantum fluctuations tend to lower it. We consider the case $M_{S}>2 m$, thus no pole below threshold is found and we have a truly resonant state. The spectral function $d_{S}(x)$ of the scalar field $S$ related to the imaginary part of the propagator is:

$$
d_{S}(x)=\frac{2 x}{\pi}\left|\lim _{\varepsilon \rightarrow 0} \operatorname{Im}\left[\Delta_{S}(x)\right]\right| \stackrel{M_{S} \geq 2 m}{=} \frac{2 x}{\pi} \frac{I_{S}(x)}{\left(x^{2}-M_{0}^{2}+R_{S}(x)\right)^{2}+I_{S}(x)^{2}} .
$$




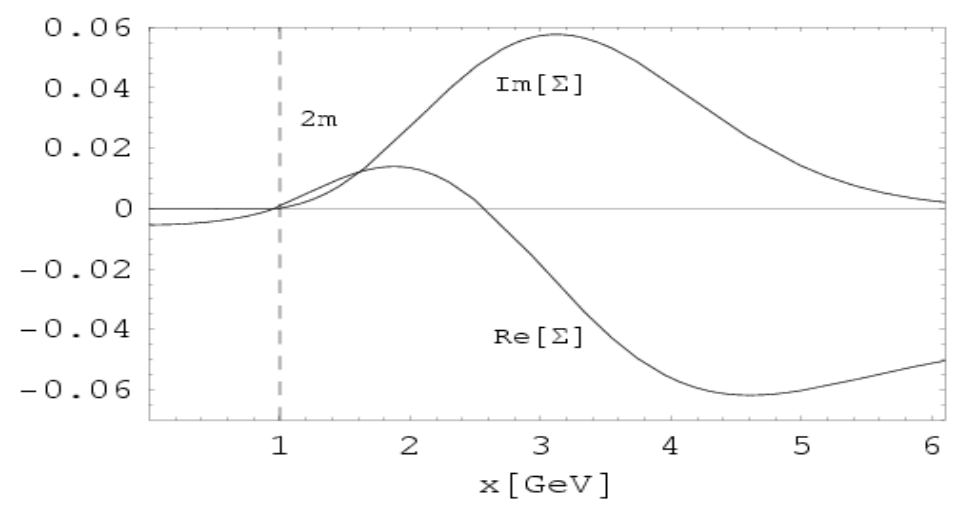

FIG. 2: Real and imaginary parts of the self-energy $\Sigma(x)$ for the illustrative values $m=0.5 \mathrm{GeV}$ and $\Lambda=1.5 \mathrm{GeV}$.

In the limit $g \rightarrow 0$ we obtain the desired spectral function $d_{S}(x)=\delta\left(x-M_{0}\right)$. The normalization of $d_{S}(x)$, i.e. the validity of the Källen-Lehman representation [30], holds for a large range of $g: \int_{0}^{\infty} d_{S}(x) d x=1$. The decay rate for the process $S \rightarrow \varphi \varphi$, which includes finite-width effects, can be defined as $\Gamma_{S \varphi \varphi}=\int_{0}^{\infty} d x d_{S}(x) \Gamma_{S \varphi \varphi}^{\mathrm{t}-1}(x)$. This formula reduces to the tree-level amplitude $\Gamma_{S \varphi \varphi}^{\mathrm{t}-1}\left(M_{0}\right)$ of Eq. (4) in the limit of small $g: \Gamma_{S \varphi \varphi}^{\mathrm{t}-1}\left(M_{0}\right) \simeq \Gamma_{S \varphi \varphi}$ for $g \rightarrow 0$. We shall however not use this formula in the present work: in fact, while mathematically correct, it presents some practical mismatch in the case of derivative couplings because a long-tail of the function $d_{S}(x) \Gamma_{S \varphi \varphi}^{\mathrm{t}-1}(x)$ may arise at large $x$. We shall therefore not compare integrated decay widths but decay amplitudes evaluated on shell, which are free form these ambiguities.

As a last step of this subsection we study the case of derivative interactions of the scalar field $S$ with two different particles $\varphi_{1}$ and $\varphi_{2}$ with masses $m_{1}$ and $m_{2}: \mathcal{L}_{i n t}=g S \partial_{\mu} \varphi_{1} \partial^{\mu} \varphi_{2}$ and its nonlocal extension. The previous formulas change as follows:

$$
\Gamma_{S \varphi_{1} \varphi_{2}}^{\mathrm{t}-1}\left(M_{0}\right)=\frac{q_{\varphi_{1} \varphi_{2}}\left(M_{0}\right)}{8 \pi M_{0}^{2}}\left[g\left(\frac{M_{0}^{2}-m_{1}^{2}-m_{2}^{2}}{2}\right) f_{\Lambda}(0, \vec{q})\right]^{2} \theta\left(M_{0}-m_{1}-m_{2}\right)
$$

where $q_{\varphi_{1} \varphi_{2}}\left(M_{0}\right)=\frac{1}{2 M_{0}} \sqrt{M_{0}^{4}+\left(m_{1}^{2}-m_{2}^{2}\right)^{2}-2\left(m_{1}^{2}+m_{2}^{2}\right) M_{0}^{2}}$. The corresponding loop function is denoted as $\widetilde{\Sigma}\left(x, m_{1}, m_{2}\right)$ and reads

$$
\widetilde{\Sigma}\left(x, m_{1}, m_{2}\right)=-i \int \frac{d^{4} q}{(2 \pi)^{4}} \frac{\left[\left(q_{1} \cdot q_{2}\right) f_{\Lambda}\left(q^{0}, \vec{q}\right)\right]^{2}}{\left[q_{1}^{2}-m_{1}^{2}+i \varepsilon\right]\left[q_{2}^{2}-m_{2}^{2}+i \varepsilon\right]} .
$$

\section{B. The $f_{0}(980)$ case}

\section{Derivative interaction with pions and kaons}

The field $f_{0}$, describing the resonance $f_{0}(980)$, interacts via derivative couplings with pions and kaons. In the local limit one has:

$$
\mathcal{L}_{i n t, f_{0}}=c_{f_{0} \pi \pi} f_{0}\left(\partial_{\mu} \vec{\pi}\right)^{2}+c_{f_{0} K K} f_{0}\left(\left(\partial_{\mu} K^{+}\right)\left(\partial^{\mu} K^{-}\right)+\left(\partial_{\mu} K^{0}\right)\left(\partial^{\mu} \bar{K}^{0}\right)\right)
$$

where $c_{f_{0} \pi \pi}$ and $c_{f_{0} K K}$ are the coupling constants. The nonlocal case, which is used in the following calculations, is obtained by delocalizing the previous interaction Lagrangian:

$$
\mathcal{L}_{i n t, f_{0}}=g f_{0}(x) \int \mathrm{d}^{4} \mathrm{y} \partial_{\mu} \vec{\pi}(x+y / 2) \partial^{\mu} \vec{\pi}(x-y / 2) \Phi(y)+\text { 'kaon part'. }
$$


The tree-level decay formulas, as function of the mass $x$, read:

$$
\begin{gathered}
\Gamma_{f_{0} \pi \pi}^{\mathrm{t}-\mathrm{l}}(x)=\frac{q_{\pi \pi}(x)}{8 \pi x^{2}}\left[A_{f_{0} \pi \pi}(x)\right]^{2} \theta\left(x-2 m_{\pi}\right) ; A_{f_{0} \pi \pi}(x)=\sqrt{6} c_{f_{0} \pi \pi}\left(\frac{x^{2}-2 m_{\pi}^{2}}{2}\right) f_{\Lambda}\left(q_{\pi \pi}(x)\right) \\
\Gamma_{f_{0} K K}^{\mathrm{t}-1}(x)=\frac{q_{K K}(x)}{8 \pi x^{2}}\left[A_{f_{0} K K}(x)\right]^{2} \theta\left(x-2 m_{K}\right) ; A_{f_{0} K K}(x)=\sqrt{2} c_{f_{0} K K}\left(\frac{x^{2}-2 m_{K}^{2}}{2}\right) f_{\Lambda}\left(q_{K K}(x)\right)
\end{gathered}
$$

where for future use we introduced the amplitudes $A_{f_{0} \pi \pi}(x)$ and $A_{f_{0} K K}(x)$. The field $f_{0}$ is dressed by pions and kaons (Fig. 1,b). Thus, the real and imaginary terms of the self-energy include contributions from both loops:

$$
\begin{gathered}
I_{f_{0}}(x)=\left(\sqrt{6} c_{f_{0} \pi \pi}\right)^{2} \operatorname{Im}\left[\Sigma\left(x, m_{\pi}\right)\right]+\left(\sqrt{2} c_{f_{0} K K}\right)^{2} \operatorname{Im}\left[\Sigma\left(x, m_{K}\right)\right], \\
R_{f_{0}}(x)=\left(\sqrt{6} c_{f_{0} \pi \pi}\right)^{2} \operatorname{Re}\left[\Sigma\left(x, m_{\pi}\right)\right]+\left(\sqrt{2} c_{f_{0} K K}\right)^{2} \operatorname{Re}\left[\Sigma\left(x, m_{K}\right)\right] .
\end{gathered}
$$

The optical theorem holds for the single channels:

$$
\left(\sqrt{6} c_{f_{0} \pi \pi}\right)^{2} \operatorname{Im}\left[\Sigma\left(x, m_{\pi}\right)\right]=x \Gamma_{f_{0} \pi \pi}^{\mathrm{t}-1}(x), \quad\left(\sqrt{2} c_{f_{0} K K}\right)^{2} \operatorname{Im}\left[\Sigma\left(x, m_{K}\right)\right]=x \Gamma_{f_{0} K K}^{\mathrm{t}-1}(x) .
$$

The spectral function $d_{f_{0}}(x)$ is just as in Eq. (7) upon setting $S=f_{0}$.

$$
\text { 2. } \phi \rightarrow \gamma \pi^{0} \pi^{0} \text { decay }
$$

Be $F^{\mu \nu}=\partial^{\mu} A^{\nu}-\partial^{\nu} A^{\mu}$ the electromagnetic field strength and $V_{\mu \nu}=\partial_{\mu} \phi_{\nu}-\partial_{\nu} \phi_{\mu}$ the field strength related to the vector field $\phi_{\mu}$, which describes the resonance $\phi(1024)$ of the PDG [31]. The Lagrangian which describes the process $\phi \rightarrow \gamma \pi^{0} \pi^{0}$ and the corresponding tree-level decay rate read

$$
\mathcal{L}_{\phi \gamma f_{0}}=c_{\phi \gamma f_{0}} f_{0} F^{\mu \nu} V_{\mu \nu} \rightarrow \Gamma_{\phi \gamma f_{0}}^{\mathrm{t}-1}(x)=c_{\phi \gamma f_{0}}^{2} \frac{\left(m_{\phi}^{2}-x^{2}\right)^{3}}{24 \pi m_{\phi}^{3}} .
$$

When $f_{0}$ is on shell one sets $x=M_{f_{0}}$. However, we are interested to the subsequent decay of $f_{0}$ into $\pi^{0} \pi^{0}$, in which $f_{0}$ is a virtual state as depicted in Fig. 1.c. The partial decay rate, defining the line shape of the $\phi \rightarrow \gamma \pi^{0} \pi^{0}$ decay, reads

$$
\frac{d \Gamma_{\phi \gamma \pi^{0} \pi^{0}}(x)}{d x}=\Gamma_{\phi \gamma f_{0}}^{\mathrm{t}-1}(x)\left[\frac{2 x}{\pi} \frac{x \Gamma_{f_{0} \pi^{0} \pi^{0}}^{\mathrm{t}-1}(x)}{\left(x^{2}-M_{0, f_{0}}^{2}+R_{f_{0}}(x)\right)^{2}+I_{f_{0}}(x)^{2}}\right]
$$

where $\Gamma_{f_{0} \pi^{0} \pi^{0}}^{\mathrm{t}-1}(x)=\frac{1}{3} \Gamma_{f_{0} \pi \pi}^{\mathrm{t}-\mathrm{l}}(x)$. It can be also rewritten as

$$
d \Gamma_{\phi \gamma \pi^{0} \pi^{0}}(x)=\Gamma_{\phi \gamma f_{0}}^{\mathrm{t}-1}(x)\left[d_{f_{0}}(x) d x\right]\left[\frac{\Gamma_{f_{0} \pi^{0} \pi^{0}}^{\mathrm{t}-\mathrm{l}}(x)}{\Gamma_{f_{0} \pi \pi}^{\mathrm{t}-\mathrm{l}}(x)+\Gamma_{f_{0} K K}^{\mathrm{t}-\mathrm{l}}(x)}\right],
$$

whose interpretation is straightforward: $\Gamma_{\phi \gamma f_{0}}^{\mathrm{t}-1}(x)$ describes the decay rate for $\phi \rightarrow \gamma f_{0}, d_{f_{0}}(x) d x$ represents the probability that the particle $f_{0}$ has a mass between $x$ and $x+d x$ and finally $\left[\Gamma_{f_{0} \pi^{0} \pi^{0}}^{\mathrm{t}-1}(x) /\left(\Gamma_{f_{0} \pi \pi}^{\mathrm{t}-1}(x)+\Gamma_{f_{0} K K}^{\mathrm{t}-\mathrm{l}}(x)\right)\right]$ describes the branching ratio of $f_{0}$ decaying into a $\pi^{0} \pi^{0}$ pair. The quantity $d \Gamma_{\phi \gamma \pi^{0} \pi^{0}}(x) / d x$ can be directly compared with experiments as we do in the next section [32].

\section{The $a_{0}(980)$ case}

\section{Derivative interaction with $\pi^{0} \eta$ and kaons}

The discussion concerning the resonance $a_{0}(980)$ follows the same line of the previous subsection. The interaction Lagrangian for the neutral field $a_{0}^{0} \equiv a_{0}^{0}(980)$ reads in the local limit

$$
\mathcal{L}_{i n t, a_{0}^{0}}=c_{a_{0} \pi \eta} a_{0}^{0}\left(\partial_{\mu} \pi^{0}\right)\left(\partial_{\mu} \eta\right)+c_{a_{0} K K} a_{0}^{0}\left(\left(\partial_{\mu} K^{+}\right)\left(\partial^{\mu} K^{-}\right)+\left(\partial_{\mu} K^{0}\right)\left(\partial^{\mu} \bar{K}^{0}\right)\right)
$$


where the coupling constants $c_{a_{0} \pi \eta}$ and $c_{a_{0} K K}$ have been introduced. The tree-level decay rates in the nonlocal case are

$$
\begin{gathered}
\Gamma_{a_{0} \pi \eta}^{\mathrm{t}-1}(x)=\frac{q_{\pi \eta}(x)}{8 \pi x^{2}}\left[A_{a_{0} \pi \eta}(x)\right]^{2} \theta\left(x-m_{\pi}-m_{\eta}\right) ; A_{a_{0} \pi \eta}(x)=c_{a_{0} \pi \eta}\left(\frac{x^{2}-m_{\pi}^{2}-m_{K}^{2}}{2}\right) f_{\Lambda}\left(q_{\pi \eta}(x)\right) \\
\Gamma_{a_{0} K K}^{\mathrm{t}-1}(x)=\frac{q_{K K}(x)}{8 \pi x^{2}}\left[A_{a_{0} K K}(x)\right]^{2} \theta\left(x-2 m_{K}\right) ; A_{a_{0} K K}(x)=\sqrt{2} c_{a_{0} K K}\left(\frac{x^{2}-2 m_{K}^{2}}{2}\right) f_{\Lambda}\left(q_{K K}(x)\right)
\end{gathered}
$$

where the amplitudes $A_{a_{0} \pi \eta}(x)$ and $A_{a_{0} K K}(x)$ have been introduced. The real and imaginary parts of the loop include contributions from $\pi \eta$ and $K K$ loops:

$$
\begin{aligned}
I_{a_{0}}(x) & =\left(c_{a_{0} \pi \eta}\right)^{2} \operatorname{Im}\left[\widetilde{\Sigma}\left(x, m_{\pi}, m_{\eta}\right)\right]+\left(\sqrt{2} c_{a_{0} K K}\right)^{2} \operatorname{Im}\left[\Sigma\left(x, m_{K}\right)\right] \\
R_{a_{0}}(x) & =\left(c_{a_{0} \pi \eta}\right)^{2} \operatorname{Re}\left[\widetilde{\Sigma}\left(x, m_{\pi}, m_{\eta}\right)\right]+\left(\sqrt{2} c_{a_{0} K K}\right)^{2} \operatorname{Re}\left[\Sigma\left(x, m_{K}\right)\right] .
\end{aligned}
$$

The spectral function $d_{a_{0}}(x)$ reads as in Eq. (7) upon setting $S=a_{0}$.

$$
\text { 2. } \phi \rightarrow \gamma \pi^{0} \eta \text { decay }
$$
by:

The interaction Lagrangian describing the process $\phi \rightarrow \gamma \pi^{0} \eta$ and the corresponding tree-level decay rate are given

$$
\mathcal{L}_{\phi \gamma a_{0}}=c_{\phi \gamma a_{0}} a_{0}^{0} F^{\mu \nu} V_{\mu \nu} \rightarrow \Gamma_{\phi \gamma a_{0}}^{\mathrm{t}-1}(x)=c_{\phi \gamma a_{0}}^{2} \frac{\left(m_{\phi}^{2}-x^{2}\right)^{3}}{24 \pi m_{\phi}^{3}}
$$

where $x=M_{a_{0}}$ for an on-shell decay. However, the $a_{0}^{0}$ meson decays subsequently into $\pi^{0} \eta$ as depicted in Fig. 1.c. As a result the line shape of the reaction $\phi \rightarrow \gamma \pi^{0} \eta$ reads:

$$
\frac{d \Gamma_{\phi \gamma \pi^{0} \eta}(x)}{d x}=\Gamma_{\phi \gamma a_{0}}^{\mathrm{t}-1}(x)\left[\frac{2 x}{\pi} \frac{x \Gamma_{a_{0} \pi \eta}^{\mathrm{t}-1}(x)}{\left(x^{2}-M_{0, a_{0}}^{2}+R_{a_{0}}(x)\right)^{2}+I_{a_{0}}(x)^{2}}\right] .
$$

\section{FIT OF THE LINE SHAPES}

\section{A. The $\phi \rightarrow \gamma \pi^{0} \pi^{0}$ fit}

Five parameters $\Lambda, M_{f_{0}}, c_{\phi \gamma f_{0}}, c_{f_{0} \pi \pi}, c_{f_{0} K K}$ determine the $\phi \rightarrow \gamma \pi^{0} \pi^{0}$ line shape via a virtual $f_{0}(980)$ meson [33]. We fix the cutoff $\Lambda=1.5 \mathrm{GeV}$; a mild dependence of the results is seen by varying $\Lambda$. Being the coupling to kaons of particular theoretical interest we perform the fit for different values of $c_{f_{0} K K}$. We start setting $c_{f_{0} K K}=0$; the fit is done to the experimental data of SND and KLOE collaborations [20, 21] corresponding to the black and grey dots in Fig. 3 retaining the data points above $0.6 \mathrm{GeV}$. The solid line is the corresponding theoretical curve for $c_{f_{0} K K}=0$, whose fit parameters are listed in the first entry of Table I. The peak of the $f_{0}$ line shape is a feature which is easily reproduced by derivative interactions.

Let us now investigate how the fit changes by increasing $c_{f_{0} K K}$ from 0 to a maximum value of $14 \mathrm{GeV}^{-1}$. As we can see in Table【, the fitted mass varies slowly from $M_{f_{0}} \sim 981 \mathrm{MeV}$ to $M_{f_{0}} \sim 971 \mathrm{MeV}, c_{f_{0} \pi \pi}$ increases from 1.28 to $2.90 \mathrm{GeV}^{-1}$ and the $\frac{\chi^{2}}{\text { d.o.f. }}$ also increases from 1.7 to 4 . We also present the on-shell amplitudes $A_{f_{0} \pi \pi}=A_{f_{0} \pi \pi}\left(M_{f_{0}}\right)$ and $A_{f_{0} K K}=A_{f_{0} K K}\left(M_{f_{0}}\right)$ defined in Eq. (12)-(13), which in the following will be compared with the results of [24]. The value of $\chi^{2} /$ d.o. $f$ increases by increasing $c_{f_{0} K K}$. However, as already mentioned in the Introduction, the coupling constant $c_{f_{0} K K}$ appears only in the denominator of the propagator of the $f_{0}$ meson (see Eq. (18)) and therefore its determination is difficult. Nevertheless, we notice that rather acceptable fits are found when varying $c_{f_{0} K K}$ in such large range. We thus not intend to state that $c_{f_{0} K K}$ is small (what would be in conflict with data from $\pi \pi$ scattering) but only that its determination from the line shape is problematic when mechanism a.1 (whose amplitude is proportional to $c_{f_{0} \pi \pi}$ ) is regarded as dominant. 


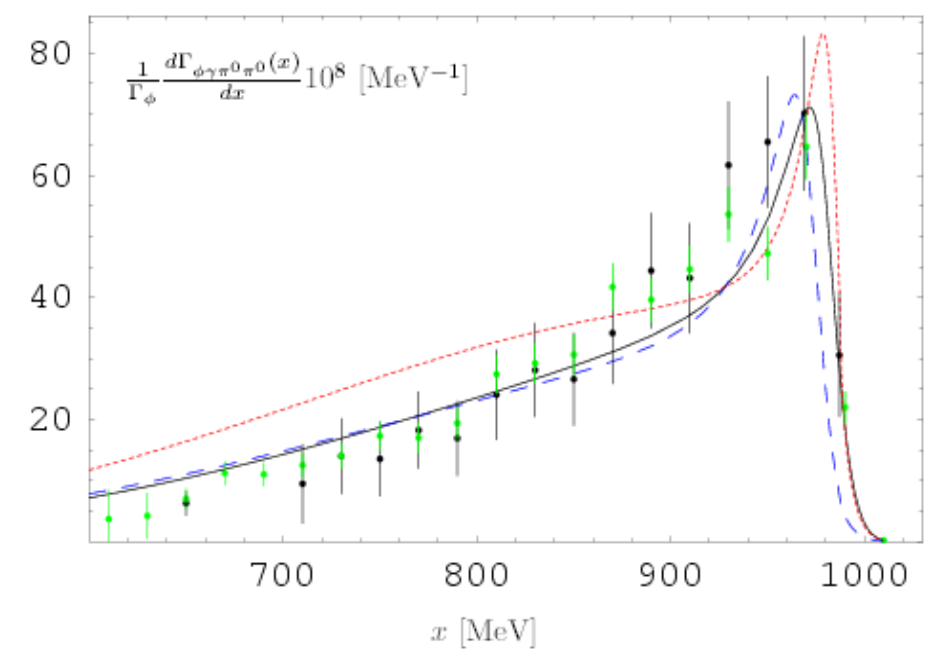

FIG. 3: Branching ratio $\frac{1}{\Gamma_{\phi}} \frac{d \Gamma_{\phi \gamma \pi^{0} \pi^{0}}(x)}{d x} 10^{8}\left[\mathrm{MeV}^{-1}\right]$ as function of the invariant mass $x$ [colors online]. $\Gamma_{\phi}=4.26 \mathrm{MeV}$ is the full width of the $\phi$ meson. We consider data sets from the SND and KLOE collaborations [20, 21] corresponding respectively to the black and grey (green online) dots. The continuous line is the result of the fit by setting $c_{f_{0} K K}=0$, the dashed line corresponds to the case $c_{f_{0} K K}=12 \mathrm{GeV}^{-1}$. Both cases are in Table I. The dotted line corresponds also to $c_{f_{0} K K}=12 \mathrm{GeV}^{-1}$ but only data points above $0.8 \mathrm{GeV}$ are used in the fit, see Table II.

TABLE I: Fit of the $\phi \rightarrow \gamma \pi^{0} \pi^{0}$ line shape data from $x>600 \mathrm{MeV}$.

\begin{tabular}{|c|c|c|c|c|c|c|}
\hline $\begin{array}{c}c_{f_{0} K K} \\
\left(\mathrm{GeV}^{-1}\right)\end{array}$ & $\begin{array}{c}M_{f_{0}} \\
(\mathrm{MeV})\end{array}$ & $\begin{array}{c}c_{f_{0} \pi \pi} \\
\left(\mathrm{GeV}^{-1}\right)\end{array}$ & $\begin{array}{c}c_{\phi \gamma f_{0}} \\
\left(\mathrm{GeV}^{-1}\right)\end{array}$ & $\frac{\chi^{2}}{d . o . f .}$ & $\begin{array}{c}A_{f_{0} K K} \\
(\mathrm{GeV})\end{array}$ & $\begin{array}{c}A_{f_{0} \pi \pi} \\
(\mathrm{GeV})\end{array}$ \\
\hline 0 & $981.8 \pm 2.8$ & $1.28 \pm 0.04$ & $0.249 \pm 0.008$ & 1.7 & 0 & $1.31 \pm 0.042$ \\
\hline 2 & $981.7 \pm 2.8$ & $1.30 \pm 0.04$ & $0.260 \pm 0.008$ & 1.9 & $0.675 \pm 0.01$ & $1.34 \pm 0.045$ \\
\hline 4 & $981.7 \pm 2.8$ & $1.38 \pm 0.05$ & $0.291 \pm 0.010$ & 2.3 & $1.35 \pm 0.01$ & $1.42 \pm 0.06$ \\
\hline 8 & $974.4 \pm 2.6$ & $2.00 \pm 0.06$ & $0.312 \pm 0.04$ & 3.4 & $2.62 \pm 0.03$ & $2.02 \pm 0.06$ \\
\hline 12 & $971.8 \pm 2.3$ & $2.59 \pm 0.06$ & $0.383 \pm 0.011$ & 3.9 & $3.89 \pm 0.04$ & $2.61 \pm 0.06$ \\
\hline 14 & $971.2 \pm 2.3$ & $2.90 \pm 0.07$ & $0.425 \pm 0.11$ & 4.0 & $4.53 \pm 0.04$ & $2.92 \pm 0.07$ \\
\hline
\end{tabular}

Notice also that at low $x$ the resonance $f_{0}(600)$ contributes to the total branching ratio as found in Refs. [17, 26]. For this reason we repeated the analysis by retaining only the data points above $800 \mathrm{MeV}$ where $f_{0}(600)$ is less relevant. The corresponding results are presented in Table II, where significantly smaller $\chi^{2}$ are obtained. Also for large values of $c_{f_{0} K K}$ the results are satisfying. As we increase $c_{f_{0} K K}$ from 0 to $14 \mathrm{GeV}^{-1}$, the fitted mass is almost unchanged $M_{f_{0}} \sim 984 \mathrm{MeV}, c_{f_{0} \pi \pi}$ increases from 1.35 to $2.35 \mathrm{GeV}^{-1}$ and the $\frac{\chi^{2}}{\text { d.o.f. }}$ increases from 0.9 to 2 . In Fig. 3 the dotted line corresponds to the case $c_{f_{0} K K}=12 \mathrm{GeV}^{-1}$ of Table II while the data above $0.8 \mathrm{GeV}$ are well described, an overestimation of data point between 0.6 and $0.8 \mathrm{GeV}$ is clearly visible. Thus, in the present analysis a destructive interference with $\sigma$ meson should occur: this fact can represent a constraint on models of light scalar mesons. Interestingly, a destructive interference of $f_{0}(600)$ and $f_{0}(980)$ is also the outcome of Ref. [21], where the $f_{0}(980)$ channel overestimates the data below $\sim 700 \mathrm{MeV}$. Nevertheless a more refined analysis including the interference with the $f_{0}(600)$ meson and using the new data of KLOE [34] -for which at present no tables with branching ratios have been presented- will be compulsory and represents an outlook of the present work. At this stage a preliminary comparison with the new data of KLOE can be done only by considering the integrated branching ratio given in Ref. [34]: $B R\left(\phi \rightarrow \pi^{0} \pi^{0} \gamma\right)=1.07 \times 10^{-4}$. Our value runs from $1.06-1.10 \times 10^{-4}$, depending on the choice for $c_{f_{0} K K}$, and therefore is consistent with the latest experimental results.

We now compare and discuss our amplitudes extracted from the KLOE and SND data with the amplitudes extracted from the experimental analyses of [24] for the $f_{0}$ meson via $j / \psi$ decay at BES. Let us stress that a comparison by using the decay widths would be less reliable, since they depend on the adopted way to evaluate them. The amplitudes 
TABLE II: Fit of the $\phi \rightarrow \gamma \pi^{0} \pi^{0}$ line shape data from $x>800 \mathrm{MeV}$.

\begin{tabular}{|c|c|c|c|c|c|c|}
\hline $\begin{array}{c}c_{f_{0} K K} \\
\left(\mathrm{GeV}^{-1}\right)\end{array}$ & $\begin{array}{c}M_{f_{0}} \\
(\mathrm{MeV})\end{array}$ & $\begin{array}{c}c_{f_{0} \pi \pi} \\
\left(\mathrm{GeV}^{-1}\right)\end{array}$ & $\begin{array}{c}c_{\phi \gamma f_{0}} \\
\left(\mathrm{GeV}^{-1}\right)\end{array}$ & $\frac{\chi^{2}}{d . o . f .}$ & $\begin{array}{c}A_{f_{0} K K} \\
(\mathrm{GeV})\end{array}$ & $\begin{array}{c}A_{f_{0} \pi \pi} \\
(\mathrm{GeV})\end{array}$ \\
\hline 0 & $984.2 \pm 3.2$ & $1.35 \pm 0.04$ & $0.263 \pm 0.007$ & 0.9 & 0 & $1.39 \pm 0.04$ \\
\hline 2 & $984.1 \pm 3.0$ & $1.38 \pm 0.04$ & $0.275 \pm 0.007$ & 0.9 & $0.68 \pm 0.01$ & $1.42 \pm 0.04$ \\
\hline 4 & $984.0 \pm 2.8$ & $1.48 \pm 0.05$ & $0.309 \pm 0.009$ & 1.0 & $1.36 \pm 0.01$ & $1.52 \pm 0.05$ \\
\hline 8 & $983.6 \pm 2.4$ & $1.81 \pm 0.07$ & $0.419 \pm 0.013$ & 1.5 & $2.72 \pm 0.03$ & $1.86 \pm 0.07$ \\
\hline 12 & $983.4 \pm 2.4$ & $2.25 \pm 0.09$ & $0.558 \pm 0.019$ & 1.9 & $4.07 \pm 0.04$ & $2.32 \pm 0.09$ \\
\hline 14 & $983.3 \pm 2.3$ & $2.50 \pm 0.11$ & $0.632 \pm 0.02$ & 2.0 & $4.75 \pm 0.04$ & $2.57 \pm 0.11$ \\
\hline
\end{tabular}

extracted in Refs. 24, 26] are :

$$
A_{f_{0} \pi \pi}=2.88 \pm 0.22 \mathrm{GeV}, A_{f_{0} K K}=5.91 \pm 0.77 \mathrm{GeV} .
$$

Our last entries in Tables $\llbracket$ and $\amalg$ are in qualitative agreement with Eq. (27). A more quantitative check is possible: we deduce from Eq. (27) the couplings $c_{f_{0} \pi \pi}$ and $c_{f_{0} K K}$ (at a fixed value of the mass of the meson) and use them in the fit of the KLOE and SND data leaving the coupling $c_{\phi \gamma f_{0}}$ as the only free parameter. The fit turns out to be acceptable with $\frac{\chi^{2}}{\text { d.o.f. }} \sim 2.4$, the extracted values of the couplings are $c_{f_{0} \pi \pi}=2.79 \mathrm{GeV}^{-1}$ and $c_{f_{0} K K}=17.37 \mathrm{GeV}^{-1}$ for $M_{f_{0}}=984 \mathrm{MeV}$. The fact that the line shape of $\phi \rightarrow \gamma \pi^{0} \pi^{0}$ can be well described by using Eq. (27) as an input shows that the BES results of Ref. [24] are compatible with our analysis.

\section{B. The $\phi \rightarrow \gamma \pi^{0} \eta$ fit}

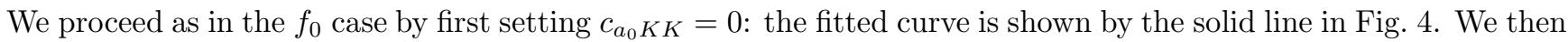
increase $c_{a_{0} K K}$ up to $14 \mathrm{GeV}^{-1}$ : the fit turns out to be satisfying in the whole range, see Table III where the mass, the couplings and the on-shell amplitudes $A_{a_{0} \pi \eta}=A_{a_{0} \pi \eta}\left(M_{a_{0}}\right), A_{a_{0} K K}=A_{a_{0} K K}\left(M_{a_{0}}\right)$ defined in Eq. (21)-(22), are reported. In Fig. 4 we also show the fitted curve in the case $c_{a_{0} K K}=12 \mathrm{GeV}^{-1}$ (dashed line). The narrow peak due to threshold effects is remarkable, but the data are not precise enough to determine its existence.

A comment about the behavior of the $\frac{\chi^{2}}{\text { d.o.f. }}$ as $c_{a_{0} K K}$ varies is in order: the fact that the fit is quite good for all the values of $c_{a_{0} K K}$ is a signal of a strong correlation between the parameters. As in the $f_{0}$ case $c_{a_{0} K K}$ only appears in the denominator of the propagator, thus the dependence of the line shape on this parameter is weak and its determination hard. While the value of the mass is almost constant, $c_{a_{0} \pi \eta}$ and $c_{\phi \gamma a_{0}}$ change significantly. This means that, while the fitted curve is in agreement with the data, the determination of the parameters is not reliable. This is due to the large error bars of the data, which in turn produce also large errors associated with each of the parameters of the fit. Again a new analysis with the new data of KLOE would be extremely important. As before a comparison with the new results of KLOE on the integrated branching ratio is useful. In Ref. 34] it is found that $B R\left(\phi \rightarrow \eta \pi^{0} \gamma\right)=6.92-7.19 \times 10^{-5}$ (respectively for the chains $\eta \rightarrow \gamma \gamma$ and $\eta \rightarrow \pi^{+} \pi^{-} \pi^{0}$ ). Our value runs from $7.37-7.73 \times 10^{-5}$, depending on the choice for $c_{a_{0} K K}$, and therefore is in reasonable agreement with the latest experimental results.

We now compare our results with the amplitudes extracted from the experimental analysis of the Crystal Barrel data of Ref. [25]:

$$
A_{a_{0} \pi \eta}=3.33 \pm 0.15 \mathrm{GeV}, A_{a_{0} K K}=3.59 \pm 0.44 \mathrm{GeV},
$$

which are not far from the last three entries of Table III. As done previously, a more quantitative test consists in deducing the couplings $c_{a_{0} \pi \eta}$ and $c_{a_{0} K K}$ from Eq. (28) and, at a fixed value of the mass, perform the fit to SND and KLOE data with only $c_{\phi \gamma a_{0}}$ as free parameter. The fit turns out to reproduce correctly the data with $\frac{\chi^{2}}{d . o . f .} \sim 1.2$, the extracted values of the couplings are $c_{a_{0} \pi \eta}=10.17 \mathrm{GeV}^{-1}$ and $c_{a_{0} K K}=9.79 \mathrm{GeV}^{-1}$ for $M_{a_{0}}=1004 \mathrm{MeV}$. Also in this case we obtain that our analysis is compatible with experimental results of a different source. 


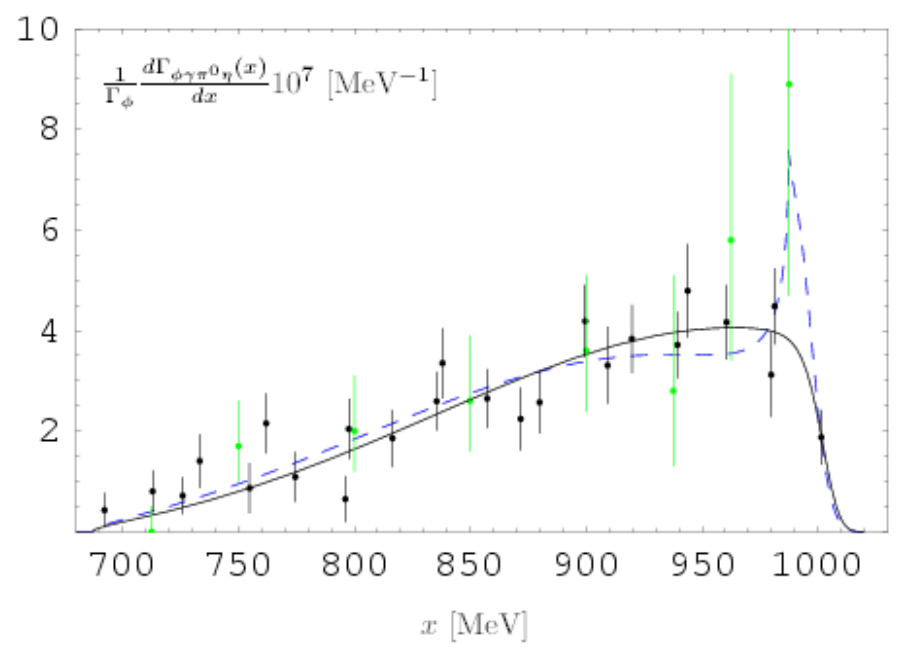

FIG. 4: Branching ratio $\frac{1}{\Gamma_{\phi}} \frac{d \Gamma_{\phi \gamma \pi^{0} \eta}(x)}{d x} 10^{7}\left[\mathrm{MeV}^{-1}\right]$ as function of the invariant mass $x$ [colors online]. $\Gamma_{\phi}=4.26 \mathrm{MeV}$ is the full width of $\phi$ meson. Grey points (green online) from [22] and black ones from [23]. The solid line corresponds to $c_{a_{0}} K K=0$ while the dashed one -with the pronounced peak at threshold- to $c_{a_{0} K K}=12 \mathrm{GeV}^{-1}$.

\section{Comparison with the fit without derivatives}

As we have seen a structureless approach using derivative couplings describes well the KLOE and SND data of the $\phi$ radiative decays. This is manly due to the fact that within the derivative approach one has an extra dependence of the form $\left(x^{2}-m_{1}^{2}-m_{2}^{2}\right)^{2}$ in the numerator of the line shape, which emphasizes the peak of the distribution at large values of the invariant mass. Since this term is not present in the non-derivative case the description of the peaked line shapes more difficult. As a comparison we also discuss in the following the results obtained when non-derivative interactions are employed. One could still obtain the peaks with non-derivative couplings by using very small decay amplitudes, which however are not in agreement with the large amplitudes extracted from Refs. [24, 25], reported in Eqs. (27) and (28).

More specifically, the inconsistency of the use of non-derivative couplings is evident in the case of the $f_{0}$ meson: when setting $c_{f_{0} K K}=c_{f_{0} \pi \pi}=0$ and allowing for nonzero $d_{f_{0} K K}$ and $d_{f_{0} \pi \pi}$ (see discussion in the Introduction) high $\frac{\chi^{2}}{\text { d.o.f. }} \gtrsim 12$ are obtained for fits above $800 \mathrm{MeV}$.

When setting in the $a_{0}$ channel $c_{a_{0} K K}=c_{a_{0} \pi \eta}=0$ and allowing for nonzero $d_{a_{0} K K}$ and $d_{a_{0} \pi \eta}$ fits with still acceptable values of $\frac{\chi^{2}}{\text { d.o.f. }} \sim 2.4$ are obtained, but the resulting amplitude $A_{a_{0} \pi \eta} \sim 1.37$ is smaller than all the cases corresponding to the derivative coupling (see Table III) and therefore the comparison with the amplitudes of Eq. (28) is problematic. Reversing the argument, we may deduce the values of $d_{a_{0} \pi \eta}$ and $d_{a_{0} K K}$ from Eq. (28) (again fixing the mass of the meson) and use them to fit the KLOE and SND data with one free coupling $c_{\phi \gamma a_{0}}$. The obtained line shape describes very badly the data with $\frac{\chi^{2}}{d . o . f .} \sim 10$, much larger than all the cases corresponding to derivative coupling. Thus, incompatibility with Eq. (28) is manifest.

All these results indicate that the use of non-derivative coupling is disfavored by present experimental informations.

\section{CONCLUSIONS}

In this work the role of derivative interactions of the mesons $f_{0}(980)$ and $a_{0}(980)$ with the pseudoscalar Goldstone bosons, which are a basic consequence of spontaneous chiral symmetry breaking as shown explicitly in chiral perturbation theory, has been investigated within a nonlocal approach in relation to the radiative decays $\phi \rightarrow \gamma \pi^{0} \pi^{0}$ and $\phi \rightarrow \gamma \pi^{0} \eta$. After developing the method at one-loop level it has been shown that a satisfactory description of the 
TABLE III: Fit of the $\phi \rightarrow \gamma \pi^{0} \eta$ line shape.

\begin{tabular}{|c|c|c|c|c|c|c|}
\hline $\begin{array}{c}c_{a_{0} K K} \\
\left(\mathrm{GeV}^{-1}\right)\end{array}$ & $\begin{array}{c}M_{a_{0}} \\
(\mathrm{MeV})\end{array}$ & $\begin{array}{c}c_{a_{0} \pi \eta} \\
\left(\mathrm{GeV}^{-1}\right)\end{array}$ & $\begin{array}{c}c_{\phi \gamma a_{0}} \\
\left(\mathrm{GeV}^{-1}\right)\end{array}$ & $\frac{\chi^{2}}{d . o . f .}$ & $\begin{array}{c}A_{a_{0} K K} \\
(\mathrm{GeV})\end{array}$ & $\begin{array}{c}A_{a_{0} \pi \eta} \\
(\mathrm{GeV})\end{array}$ \\
\hline 0 & $1005 \pm 18$ & $4.68 \pm 0.90$ & $0.263 \pm 0.046$ & 0.88 & 0 & $1.54 \pm 0.3$ \\
\hline 2 & $1005 \pm 18$ & $4.71 \pm 0.94$ & $0.278 \pm 0.050$ & 0.88 & $0.74 \pm 0.05$ & $1.55 \pm 0.32$ \\
\hline 4 & $1004 \pm 18$ & $4.80 \pm 1.07$ & $0.321 \pm 0.06$ & 0.88 & $1.47 \pm 0.10$ & $1.57 \pm 0.36$ \\
\hline 8 & $1003 \pm 18$ & $5.10 \pm 1.62$ & $0.486 \pm 0.13$ & 0.88 & $2.92 \pm 0.20$ & $1.67 \pm 0.53$ \\
\hline 12 & $1002 \pm 19$ & $5.52 \pm 2.49$ & $0.731 \pm 0.28$ & 0.92 & $4.36 \pm 0.31$ & $1.80 \pm 0.82$ \\
\hline 14 & $1001 \pm 20$ & $5.77 \pm 3.01$ & $0.873 \pm 0.400$ & 0.92 & $5.09 \pm 0.37$ & $1.88 \pm 1.00$ \\
\hline
\end{tabular}

line shapes of the reactions $\phi \rightarrow \gamma \pi^{0} \pi^{0}$ and $\phi \rightarrow \gamma \pi^{0} \eta$ is obtained: the property of derivatives interactions seems tailor-made to describe the well-marked peaks measured experimentally.

More specifically, in the $\phi \rightarrow \gamma \pi^{0} \pi^{0}$ case we fitted our theoretical curves, which make use of derivative interactions only and involve a virtual $f_{0}$ meson, to data above 600 and $800 \mathrm{MeV}$ (Fig. 3 and Tables [ and [I): in the latter case the influence of the $f_{0}(600)$ resonance is less relevant. It has been also stressed that the determination of the coupling to kaons from the line shape only is hard because the latter enters only in the propagator of the scalar meson. Interestingly, fits involving a large $f_{0} \rightarrow \bar{K} K$ coupling, as found in 24], are acceptable $\left(\chi^{2} /\right.$ d.o.f. $\left.\lesssim 2\right)$ when datapoints above $800 \mathrm{MeV}$ are considered. Notice that in this case data below $800 \mathrm{MeV}$ are overestimated as the dotted line in Fig. 3 shows. A destructive interference in the $f_{0}(600)$ channel should be invoked in order to get agreement with data, see also Ref. 21]. While leaving a more detailed study as an outlook, this fact can represent a significant constraint on models based on a particular interpretation of the scalar mesons, such as the tetraquark assignment. We also verified that the usage of non-derivative couplings generates a bad agreement with the experimental results $\left(\chi^{2} /\right.$ d.o.f. $\left.>10\right)$ : the line shape of the $f_{0}$ meson cannot be reproduced for any choice of the parameters.

In the $\phi \rightarrow \gamma \pi^{0} \eta$ case, occurring via a virtual $a_{0}$ meson, a good agreement is found (Fig. 团and Table III). However, even sizable variations of the parameters do not make the agreement worse: for this reason a determination of the parameters is hard. As above, the determination of the coupling to kaons is problematic. However, when using the amplitudes extracted from Ref. 25] to deduce the couplings with pseudoscalar mesons a good description of the line shape is obtained: this fact shows compatibility of the study of Crystal Barrel data in Ref. 25] with KLOE-SND data when derivative interactions are used (notice also the pronounced peak close to threshold of the dashed line in Fig. 4. whose appearance is due to a large coupling to kaons). On the contrary, the same procedure in the non-derivative case shows that the line shape of $\phi \rightarrow \gamma \pi^{0} \eta$ is badly reproduced.

Summarizing, the results of the present study point out that derivative-type interactions (denoted as mechanism a.1 in the Introduction) with pseudoscalar mesons can play an important role for the study of the scalar states $a_{0}(980)$ and $f_{0}(980)$. The next step of the present work is the inclusion of non-derivative interactions (explicit chiral symmetry breaking terms, mechanism a.2) besides the derivative ones. The next-to-next (more ambitious) step is the inclusion at the same time and in the same theoretical framework, together with the mechanisms a.1 (derivative couplings) and a.2 (non-derivative couplings), also of the correspondent kaon-loop driven contributions (mechanisms b.1 and b.2 respectively). To achieve these goals, which can also set up which mechanism (if any) is dominant in radiative $\phi$ decays, further work is required. The aim is a definitive determination of the relevant amplitudes, which represent a necessary tool to test models and thus to understand the nature of the light scalar mesons. To this end the use of other experimental informations from $j / \psi$ decay and Crystal Barrel data is necessary. Also, a comparison with the newest KLOE data [34] and the study of radiative decays of scalar resonances [35] represents an interesting development.

Acknowledgements We thank D. Bugg and C. Bini for useful discussions. G.P. acknowledges financial support from INFN.

[1] C. Amsler and N. A. Tornqvist, Phys. Rept. 389, 61 (2004).

[2] N. N. Achasov and V. N. Ivanchenko, Nucl. Phys. B 315 (1989) 465. M. Boglione and M. R. Pennington, Eur. Phys. J. C 30 (2003) 503 arXiv:hep-ph/0303200. F. E. Close, N. Isgur and S. Kumano, Nucl. Phys. B 389 (1993) 513 arXiv:hep-ph/9301253.

[3] F. E. Close, N. Isgur and S. Kumano, Nucl. Phys. B 389 (1993) 513 arXiv:hep-ph/9301253. 
[4] E. van Beveren, F. Kleefeld, G. Rupp and M. D. Scadron, Mod. Phys. Lett. A 17 (2002) 1673 arXiv:hep-ph/0204139. M. D. Scadron, G. Rupp, F. Kleefeld and E. van Beveren, Phys. Rev. D 69 (2004) 014010 [Erratum-ibid. D 69 (2004) 059901] arXiv:hep-ph/0309109.

[5] R. L. Jaffe, Phys. Rev. D 15 (1977) 267. R. L. Jaffe, Phys. Rev. D 15 (1977) 281. R. L. Jaffe and F. E. Low, Phys. Rev. D 19, 2105 (1979). L. Maiani, F. Piccinini, A. D. Polosa and V. Riquer, Phys. Rev. Lett. 93 (2004) 212002 arXiv:hep-ph/0407017. G. '. Hooft, G. Isidori, L. Maiani, A. D. Polosa and V. Riquer, arXiv:0801.2288 [hep-ph]. A. H. Fariborz, R. Jora and J. Schechter, Phys. Rev. D 77 (2008) 094004 arXiv:0801.2552 [hep-ph]].

[6] F. Giacosa, Phys. Rev. D 74 (2006) 014028 arXiv:hep-ph/0605191.

[7] M. B. Voloshin and L. B. Okun, JETP Lett. 23, 333 (1976) [Pisma Zh. Eksp. Teor. Fiz. 23, 369 (1976)]. K. Maltman and N. Isgur, Phys. Rev. Lett. 50, 1827 (1983). K. Maltman and N. Isgur, Phys. Rev. D 29, 952 (1984). V. Baru, J. Haidenbauer, C. Hanhart, Yu. Kalashnikova and A. E. Kudryavtsev, Phys. Lett. B 586 (2004) 53 arXiv:hep-ph/0308129. T. Branz, T. Gutsche and V. E. Lyubovitskij, arXiv:0712.0354 [hep-ph].

[8] A. H. Fariborz, R. Jora and J. Schechter, Phys. Rev. D 72 (2005) 034001. A. H. Fariborz, Int. J. Mod. Phys. A 19 (2004) 2095. M. Napsuciale and S. Rodriguez, Phys. Rev. D 70 (2004) 094043.

[9] F. Giacosa, Phys. Rev. D 75 (2007) 054007.

[10] J. R. Pelaez, Phys. Rev. Lett. 92 (2004) 102001. Mod. Phys. Lett. A 19 (2004) 2879. J. A. Oller and E. Oset, Nucl. Phys. A 620 (1997) 438 [Erratum-ibid. A 652 (1999) 407] N. Mathur et al., Phys. Rev. D 76 (2007) 114505.

[11] C. Amsler and F. E. Close, Phys. Rev. D 53 (1996) 295 arXiv:hep-ph/9507326. C. Amsler and F. E. Close, Phys. Lett. B 353, 385 (1995).

[12] W. J. Lee and D. Weingarten, Phys. Rev. D 61, 014015 (2000). F. E. Close and A. Kirk, Eur. Phys. J. C 21, 531 (2001). F. Giacosa, T. Gutsche, V. E. Lyubovitskij and A. Faessler, Phys. Rev. D 72, 094006 (2005). F. Giacosa, T. Gutsche, V. E. Lyubovitskij and A. Faessler, Phys. Lett. B 622, 277 (2005). F. Giacosa, T. Gutsche and A. Faessler, Phys. Rev. C 71, 025202 (2005) arXiv:hep-ph/0408085.

[13] G. Ecker, J. Gasser, A. Pich and E. de Rafael, Nucl. Phys. B 321 (1989) 311.

Notice however that the couplings to pseudoscalar mesons evaluated by Ecker et al. correspond to a quark-antiquark assignment for the light scalars (the parameters scale accordingly in the large $N_{c}$ series). If the light scalars are tetraquark states the Clebsch-Gordon coefficients are different and are evaluated in Ref. [5] and by one of us in Ref. [6] where also the next-to-leading order in the large $N_{c}$ expansion has been systematically evaluated. On the other hand, Eq. (1), where no assumption is made on $\mathrm{SU}(3)$ flavor relations, is valid also within a tetraquark scenario.

[14] D. Black, M. Harada and J. Schechter, Phys. Rev. D 73 (2006) 054017 arXiv:hep-ph/0601052.

[15] G. Isidori, L. Maiani, M. Nicolaci and S. Pacetti, JHEP 0605 (2006) 049 arXiv:hep-ph/0603241.

[16] N. N. Achasov and V. V. Gubin, Phys. Rev. D 56 (1997) 4084 arXiv:hep-ph/9703367. N. N. Achasov and A. V. Kiselev, Phys. Rev. D 73 (2006) 054029 [Erratum-ibid. D 74 (2006) 059902] arXiv:hep-ph/0512047.

[17] Yu. S. Kalashnikova, A. E. Kudryavtsev, A. V. Nefediev, C. Hanhart and J. Haidenbauer, Eur. Phys. J. A 24 (2005) 437 arXiv:hep-ph/0412340. H. Nagahiro, L. Roca and E. Oset, arXiv:0802.0455 [hep-ph]. J. A. Oller, Nucl. Phys. A 714 (2003) 161 arXiv:hep-ph/0205121]. A. Bramon, R. Escribano, J. L. Lucio M., M. Napsuciale and G. Pancheri, Phys. Lett. B 494 (2000) 221 arXiv:hep-ph/0008188]. E. Marco, S. Hirenzaki, E. Oset and H. Toki, Phys. Lett. B 470 (1999) 20 arXiv:hep-ph/9903217. J. E. Palomar, L. Roca, E. Oset and M. J. Vicente Vacas, Nucl. Phys. A 729 (2003) 743 arXiv:hep-ph/0306249. V. E. Markushin, Eur. Phys. J. A 8 (2000) 389 arXiv:hep-ph/0005164. Yu. M. Bystritskiy, M. K. Volkov, E. A. Kuraev, E. Bartos and M. Secansky, Phys. Rev. D 77 (2008) 054008 [arXiv:0712.0304 [hep-ph]]. T. Teshima, I. Kitamura and N. Morisita, Phys. Rev. D 76 (2007) 054002 arXiv:hep-ph/0702256.

[18] G. 't Hooft, Nucl. Phys. B 72 (1974) 461. E. Witten, Nucl. Phys. B 160 (1979) 57.

In detail, within the quarkonium assignment the $f_{0}(980)$ contains a consistent $\bar{s} s$ amount in its wave function. Then, the amplitude involving a $s$-quark loop for the process $\phi \rightarrow \gamma f_{0}$ scales as $N_{c}^{0}$ while the amplitude involving the kaon loop scales as $N_{c}^{-1}$ : this is due to the fact that a kaon loop does not carry a factor $N_{c}$, which is present in the quark-loop. In the tetraquark assignment the exact scaling factors of the amplitudes are not known, but the ratio among them is fixed: as in the quarkonium assignment and for the same reason the amplitude of the kaon loop contribution is suppressed of a factor $N_{c}^{-1}$ with respect to the quark-loop contribution. The discussion is slightly different for the $a_{0}(980)$ meson: in the quarkonium assignment both the quark and kaon loop amplitudes for the process $\phi \rightarrow \gamma a_{0}$ scale as $N_{c}^{-1}$. (In fact, the quark loop amplitude is OZI-suppressed because $\phi \simeq \bar{s} s$ and $a_{0}^{0}=\sqrt{1 / 2}(\bar{u} u+\bar{d} d)$ and does carries an extra-factor $\left.1 / N_{c}\right)$. In the tetraquark assignment -just as in the $f_{0}$ case- the kaon loop amplitude is suppressed of a factor $N_{c}^{-1}$ with respect to the quark loop contribution.

[19] F. Giacosa and G. Pagliara, Phys. Rev. C 76 (2007) 065204 arXiv:0707.3594 [hep-ph]].

[20] M. N. Achasov et al., Phys. Lett. B 485 (2000) 349 arXiv:hep-ex/0005017.

[21] A. Aloisio et al. [KLOE Collaboration], Phys. Lett. B 537 (2002) 21 arXiv:hep-ex/0204013].

[22] M. N. Achasov et al., Phys. Lett. B 479 (2000) 53 arXiv:hep-ex/0003031.

[23] A. Aloisio et al. [KLOE Collaboration], Phys. Lett. B 536 (2002) 209 arXiv:hep-ex/0204012.

[24] M. Ablikim et al. [BES Collaboration], Phys. Lett. B 607 (2005) 243 arXiv:hep-ex/0411001.

[25] D. V. Bugg, V. V. Anisovich, A. Sarantsev and B. S. Zou, Phys. Rev. D 50 (1994) 4412.

[26] D. V. Bugg, Eur. Phys. J. C 47 (2006) 57 arXiv:hep-ph/0603089. D. V. Bugg, arXiv:hep-ex/0510014.

[27] D. V. Bugg, Eur. Phys. J. C 47 (2006) 45 arXiv:hep-ex/0603023.

[28] N. A. Tornqvist, Z. Phys. C 68 (1995) 647 arXiv:hep-ph/9504372.

[29] S. Godfrey and N. Isgur, Phys. Rev. D 32 (1985) 189. 
[30] N. N. Achasov and A. V. Kiselev, Phys. Rev. D 70 (2004) 111901 arXiv:hep-ph/0405128].

[31] W. M. Yao et al. [Particle Data Group], J. Phys. G 33 (2006) 1.

[32] There is another process with the same initial and final states which can contribute to the line shape: $\phi \rightarrow \rho^{0} \pi^{0} \rightarrow \pi^{0} \pi^{0} \gamma$. This process has been included to fit the data of KLOE and SND in Refs. 20, 21], however it was found that its contribution is small. Thus, we prefer, for the sake of simplicity, to neglect this term in this preliminary investigation on the derivative interactions.

[33] Notice that within our formalism we can consider $c_{f_{0} \pi \pi}$ as a fit-parameter at variance from Ref. [14] where only the product $c_{\phi \gamma f_{0}} c_{f_{0} \pi \pi}$ appears in the theoretical curve. Therefore only $c_{\phi \gamma f_{0}}$ is used as a fit-parameter while $c_{f_{0} \pi \pi}$ is fixed.

[34] F. Ambrosino et al. [KLOE Collaboration], Eur. Phys. J. C 49 (2007) 473 arXiv:hep-ex/0609009. F. Ambrosino et al. [KLOE Collaboration], Phys. Lett. B 634 (2006) 148 arXiv:hep-ex/0511031]. F. Ambrosino et al. [KLOE Collaboration], arXiv:0707.4609 [hep-ex].

[35] Yu. Kalashnikova, A. E. Kudryavtsev, A. V. Nefediev, J. Haidenbauer and C. Hanhart, Phys. Rev. C 73 (2006) 045203 arXiv:nucl-th/0512028. D. Black, M. Harada and J. Schechter, Phys. Rev. Lett. 88 (2002) 181603 arXiv:hep-ph/0202069. M. R. Pennington, Phys. Rev. Lett. 97 (2006) 011601. F. Giacosa, T. Gutsche and V. E. Lyubovitskij, Phys. Rev. D 77 (2008) 034007 arXiv:0710.3403 [hep-ph]]. 